\section{CONFLICTOS ENTRE DERECHOS CONSTITUCIONALES Y MANERAS DE RESOLVERLOS}

\author{
José Juan Moreso \\ (Universitat Pompeu Fabra, Barcelona)
}

\section{CONFLICTS AMONG CONSTITUTIONAL RIGHTS AND WAYS TO SOLVE THEM}

\begin{abstract}
This paper deals with the question of the conflict of constitutional rights which acknowledge basic rights. It is shown that there are two extreme accounts: the subsumptive approach and the particularist approach that embody two main conceptions of practical rationality. Between both approaches there is room for a range of options. Two of them are taken into account: the proportionalist approach, which conserves the scope of rights restricting their stringency, and it is argued in favour of the specificationist approach, which preserve the stringency of rights restricting their scope.
\end{abstract}

KEY WORDS: Constitutional theory; conflicts of rights; subsumptive rationality; moral particularism.

\section{INTRODUCCIÓN: TRES CASOS CONSTITUCIONALES}

En este trabajo me propongo prestar especial atención a los problemas de aplicación de las constituciones que contienen declaraciones de derechos, dado que las pautas que establecen derechos pueden entrar en conflicto entre sí.

En este sentido, se trata de un fenómeno muy conocido en la filosofía práctica en general y en la filosofía moral en particular. Supongamos que una persona sugiere que hay un principio moral que establece "Se deben cumplir las promesas", entonces otra persona trata de mostrarle que el hecho de haber prometido hacer A no siempre constituye una razón para hacer $A$, puesto que puede chocar con otro principio moral, así ¿es una razón para matar a una persona el hecho de haberlo prometido a cambio de un millón de euros?, parece que aquí vence el principio moral con arreglo al cual no se debe matar.

Sin embargo, un modo aparentemente muy plausible de concebir la racionalidad práctica consiste en justificar una determinada acción aludiendo a un principio subyacente que, en aquellas circunstancias, requiere reali-
RESUMEN: En este trabajo se analiza la cuestión de la colisión entre principios constitucionales que reconocen derechos básicos. Se muestra que hay dos posiciones extremas: el enfoque subsuntivo y el enfoque particularista, que encarnan dos grandes concepciones de la racionalidad práctica. Entre ambos enfoques hay lugar para un amplio espectro de opciones. Se toman dos en cuenta: el enfoque proporcionalista, que conserva el alcance de los derechos restringiendo su fuerza y el enfoque especificacionista, a favor del cual se argumenta, que conserva la fuerza de los derechos restringiendo su alcance.

PALABRAS CLAVE: Teoría constitucional; conflictos de derechos; racionalidad subsuntiva; particularismo moral.

zar dicha acción. Si hubiera dos principios en conflicto, que requiriesen acciones incompatibles, este modo de concebir la racionalidad práctica sería insuficiente. Por esta razón, lo que podemos denominar el enfoque subsuntivo es atractivo, se trata de la razón que llevaba a Kant (Kant, 1989, 31) a sostener que obligaciones non collinduntur.

La aplicación del derecho, tal y como usualmente la comprendemos, es una instancia de esta concepción general de la racionalidad práctica. Aplicar el derecho consiste en determinar la norma individual que establece una cierta consecuencia normativa para un caso individual determinado. Para tal fin, se trata de mostrar que dicho caso individual es una instancia de un caso genérico al que una norma aplicable correlaciona con esa consecuencia normativa. Dicha operación se conoce con el nombre de subsunción.

Ahora bien, hay supuestos en los que el enfoque subsuntivo parece plantear problemas. Traigo aquí a colación tres de los que aparecen en los diversos ensayos de la tercera parte: el caso Titanic, el caso del cura de Hío y el caso del niño Marcos. 
El caso Titanic ${ }^{1}$ es un caso de colisión entre la libertad de expresión y el derecho al honor en el cual la revista satírica Titanic había llamado "asesino nato" $y$, en otra edición posterior, "tullido" a un oficial de la reserva que era parapléjico y que había logrado ser convocado de nuevo para llevar a cabo unos ejercicios militares. El Tribunal Superior de Dusseldorf condenó a la revista a pagar una indemnización de 12.000 marcos alemanes al oficial. La revista interpuso un recurso de amparo y el Tribunal Constitucional alemán consideró que mientras Ilamar al oficial "asesino nato" era una interferencia moderada o leve en su derecho al honor, porque este tipo de apelativos eran usuales en su estilo satírico, y en cambio la interferencia en la libertad de expresión se considera grave; llamar "tullido" a un parapléjico se considera una interferencia gravisima que derrota la interferencia grave en la libertad de expresión de la revista. 0 sea que por este segundo apelativo únicamente estimó el Tribunal el recurso de amparo.

El caso del cura de Hío es, en cambio, un supuesto de la jurisprudencia del Tribunal Constitucional español ${ }^{2}$, que en 1992 resolvió un recurso de amparo en el cual el cura de Hío demandaba al diario El País que en 1984 había publicado una noticia según la cual el cura de dicha parroquia lideraba, garrote en mano, una manifestación de vecinos contra algunos ciudadanos que practicaban el nudismo en determinadas playas gallegas. Resultó que, a pesar de la diligencia del redactor de la noticia al comprobar los datos, el cura de Hío no estaba involucrado en dicha circunstancia. ¿Cómo resolver el conflicto entre las razones constitucionales que favorecen la libertad de información y aquellas que favorecen el derecho al honor? El Tribunal Constitucional español se inclinó, esta vez, por la libertad de información, con el argumento según el cual los informadores están justificados no sólo con la verdad de las noticias que publican, sino también con su veracidad, para la que basta que la noticia, si falsa, esté diligentemente contrastada.

Y, por último, el caso del niño Marcos: el día 3 de septiembre de 1994, Marcos, A. V., un niño de 13 años que vivía en Ballobar (Huesca) con sus padres, tuvo una caída con su bicicleta a la que no dieron mayor importancia. Pocos días después, el 8 de septiembre, al haber sangrado por la nariz en varias ocasiones, fue llevado por su madre al médico que aconsejó su ingreso en el Hospital Arnau de Vilanova de Lleida. En dicho hospital, después de las pruebas per- tinentes y ante el alto riesgo de fuertes hemorragias, los médicos prescribieron una transfusión de sangre. En ese momento los padres del menor manifestaron educadamente que su religión, profesaban la religión de los testigos de Jehová, les prohibia el uso de transfusiones y preguntaron si había algún tratamiento alternativo. Los médicos afirmaron que ellos no conocían ningún tratamiento alternativo, se negaron a conceder el alta voluntaria que los padres solicitaban, y se dirigieron al Juzgado de Guardia para que autorizara la transfusión. Los padres, a pesar de no compartir dicha decisión, la acataron y no hicieron nada para impedir la transfusión, que fue autorizada por el Juzgado. Marcos, que profesaba la misma religión que sus padres, rechazó con auténtico terror la intervención, reaccionando con gran excitación y violencia que los médicos estimaron muy peligrosas, pues podian precipitar una hemorragia cerebral. Fracasados sus intentos de convencer al menor, el personal sanitario del Hospital solicitó a los padres que trataran de convencer a Marcos, a lo que los padres se negaron arguyendo que ellos, como Marcos, pensaban que Dios no autorizaba las transfusiones de sangre ni en los casos de peligro para la vida. Desestimado el uso de algún procedimiento anestésico por razones médicas, al final el Hospital accedió a otorgar el alta voluntaria para que los padres buscaran algún tratamiento alternativo en otro lugar. Regresaron a su domicilio y tres días después lo trasladaron a varios hospitales de Barcelona, donde les reiteraron la necesidad de la transfusión, pero ninguno de dichos hospitales, dada la expresa oposición de Marcos y sus padres, tomó la decisión de intentar llevarla a cabo. Regresaron a su domicilio, en el cual el menor contó solamente con la asistencia del médico titular de Ballobar, hasta que el día 14 de septiembre el Juzgado de Instrucción de Fraga (Huesca) dispuso mediante Auto, a la vista de que el menor empeoraba progresivamente por anemia aguda posthemorrágica, la autorización para la entrada en el domicilio del menor con el fin de que le fueran realizados los tratamientos médicos que precisaba, es decir, una transfusión de sangre. Marcos fue trasladado en ambulancia primero al Hospital de Barbastro y después al Hospital Miguel Servet de Zaragoza al que llegó con signos clínicos de descerebración por hemorragia cerebral. Marcos falleció el día 15 de septiembre en el Hospital Miguel Servet.

Los padres de Marcos fueron acusados del delito de homicidio, del que fueron absueltos por sentencia de la Audiencia Provincial de Huesca de 20 de noviembre de 1996. 
El Tribunal Supremo casó y anuló dicha sentencia y les condenó, por sentencia de 27 de junio de 1997, por el delito de homicidio con la circunstancia atenuante, muy cualificada, de obcecación o estado pasional, a la pena de dos años y seis meses de prisión, y al pago de las costas correspondientes. Los condenados interpusieron recurso de amparo contra dicha sentencia condenatoria ante el Tribunal Constitucional que, en STC 154/2002, de 18 de julio, resolvió conceder el amparo, declarar vulnerado el derecho fundamental a la libertad religiosa $y$, en consecuencia, anular la decisión del Tribunal Supremo.

Aquí vemos, en cambio, una colisión entre dos derechos diversos: el derecho a la vida y el derecho a la libertad religiosa.

\section{El desafío particularista al enfooue SUBSUNTIVO}

La variedad de nuestro paisaje moral hace comprensible el desafío al enfoque subsuntivo por parte de las concepciones particularistas ${ }^{3}$. Según dichas concepciones, el hecho de que una propiedad de una situación determinada sea relevante en relación con su corrección, no presupone que lo sea en cualquier otra circunstancia. Digámoslo con un ejemplo de aroma kantiano, el hecho de que una persona posea una información verdadera es una razón para afirmarla cuando se la piden ${ }^{4}$. Ahora bien, si lo que solicitan a alguien que está en posesión de la información es el escondite de una persona inocente a la que quieren asesinar, entonces el hecho de poseer dicha información no debe conducir a relevarla, porque -por así decirlo- vence el principio que obliga a proteger a las personas inocentes cuando está en nuestra mano. Pénsemos en un buen profesor universitario prusiano durante la segunda guerra mundial que, sabedor porque él mismo se lo proporcionó, del escondite de una mujer de origen judío, que había sido una de sus alumnas, revela el lugar como respuesta a una pregunta de un oficial de las SS. Tal vez por esta razón David Ross (Ross, 1930) sostuvo que los deberes morales no son absolutos, sino prima facie. Ahora bien, una vez hacemos esta concesión, ¿dónde podemos detenernos? Regresemos a nuestro ejemplo referido al cumplimiento de las promesas. Supóngamos que, ante la promesa de asesinar a alguien, se replica reformulando la norma moral propuesta para qué no incluya estos supuestos, por ejemplo, "se deben cumplir las promesas que no conllevan la realización de comportamientos inmorales". Ahora el particularista o defensor del enfoque subsuntivo vuelve a la carga: ¿qué ocurre si la promesa ha sido obtenida bajo la amenaza de secuestrar a la hija del que ha prometido? Y el universalista replica con una nueva formulación: "se deben cumplir las promesas que no han sido otorgadas bajo amenaza ni conllevan la realización de actos inmorales". El particularista todavia puede desafiar diciendo que si arrancamos de un torturador, bajo amenaza, la promesa de dejar de torturar, entonces debe dejar de torturar y el universalista puede añadir el adjetivo "injustas" al sustantivo "amenazas". Y así sucesivamente. Los que cuestionan el enfoque subsuntivo de la moralidad sostienen que no hay modo plausible de poner fin a este sucesivamente. Sostienen que los contextos se comportan de modos muy diversos y que, por lo tanto, no hay manera de atrapar un principio moral válido en todos los contextos de manera invariable.

Esta conclusión produce cierto desazón cuando se traslada a la teoría del derecho, puesto que conllevaría el corolario de que en la aplicación de las pautas que establecen los derechos constitucionales, la solución dependería siempre del contexto, dejando a los destinatarios de las normas al arbitrio de los aplicadores 5 .

\section{INTERLUDIO: UN AMPLIO ESPECTRO DE OPCIONES INTERMEDIAS}

En realidad, tanto el enfoque subsuntivo como el enfoque particularista constituyen dos extremos, que iluminan algunos aspectos de la cuestión a costa de distorsionar de manera excesiva otros aspectos. Entre estos extremos, cabe un espectro de posiciones intermedias ${ }^{6}$.

Dicho en palabras de Russ Shafer-Landau?:

Cuando nuestros derechos parecen entrar en conflicto con otras consideraciones morales, incluyendo otros derechos morales, podemos resolver la tensión reduciendo el alcance del derecho o bien reduciendo su fuerza.

Reducir la fuerza de los principios comporta considerar que los principios propiamente cubren los casos en los 
cuales varios de ellos pueden entrar en conflicto, pero en dichos supuestos uno de los principios resulta vencedor, conserva su fuerza, mientras el otro -el vencido- pierde su aplicabilidad al caso concreto. Me referiré aquí a uno de los enfoques posibles de esta concepción, el enfoque proporcionalista, tal como ha sido presentado por Robert Alexy, cuyas ideas acerca de la denominada ponderación han sido y son la posición más articulada y sugerente de la que disponemos en la teoría constitucional del presente ${ }^{8}$.

Reducir el alcance de los principios conservando su fuerza representa considerar la formulación de los principios como incompleta y expandirla de modo que, conservando el alcance, los principios debidamente reformulados no entren ya en conflicto. Se trata de la posición que subyace a todos los ensayos de esta parte y que podemos denominar enfoque especificacionista ${ }^{10}$.

\section{El ENFOQUE PROPORCIONALISTA}

Veamos, en primer lugar, la concepción proporcionalista tal como la concibe Robert Alexy. Para dicha concepción, mientras las colisiones entre normas son un síntoma de que, o bien alguna de las normas es inválida, o bien una puede considerarse una excepción de la otra; las colisiones entre principios han de ser resueltas de modo distinto. Cuando dos principios entran en colisión (por ejemplo, porque el primero establece que una conducta determinada está prohibida y el segundo que está permitida) uno de los dos ha de ceder frente al otro. Pero esto no significa que uno de los dos principios sea inválido, ni que en el principio desplazado haya que introducir alguna excepción. Lo que sucede es que, en determinadas circunstancias, un principio precede al otro. Es por esta razón que se afirma que, en los casos concretos, los principios tienen diferente peso y el conflicto ha de resolverse según la dimensión de peso y no según la dimensión de validez.

La dimensión de peso, entonces, configura el núcleo de la ponderación. Dicha operación forma parte de lo que es requerido por un principio más comprensivo: el principio de proporcionalidad. Este principio comprende tres subprincipios: a) el principio de adecuación, es decir, que el sacrificio impuesto en el ejercicio de un derecho sea adecuado para preservar otro derecho o un bien constitucionalmente protegido, b) el principio de necesidad, esto es, que el sacrificio impuesto sea necesario -que no exista otro menos lesivo- para preservar otro derecho o un bien constitucionalmente protegido"11, y c) el principio de proporcionalidad en sentido estricto, en donde la ponderación propiamente dicha ocupa su lugar, que se afecte al ejercicio del derecho en el menor grado posible compatible con la mayor satisfacción en el ejercicio del otro derecho.

Según Alexy, la ponderación puede ser dividida en tres etapas. La primera etapa comporta el establecimiento del grado de no satisfacción del primer principio. La segunda etapa consiste en establecer el grado de satisfacción del principio en pugna con el primero. Finalmente, la tercera etapa evalúa si la importancia de la satisfacción del segundo principio justifica la falta de satisfacción del primero. Podemos dividir el grado de afección a un derecho determinado en tres rangos: leve, medio y grave. Como es obvio, estos grados de afección son relativos al contexto establecido por el caso concreto. De ello resulta lo siguiente: las vulneraciones leves de un derecho fundamental ceden ante la protección media y la grave de otro derecho fundamental, y las medias ceden ante las graves. Quedan tres casos de empate, en donde -si entiendo bien la propuesta de Alexy- el legislador goza de discreción para afectar uno u otro derecho, lo que equivale a decir que, en los casos de empate, las restricciones legislativas al ejercicio de un derecho fundamental están justificadas.

Entonces, en la fórmula más simple, el peso concreto de un principio $P i$ que colisiona con otro principio $P j$, es decir, $W i, j$, es el cociente que resulta de dividir la intensidad de la interferencia en el primer principio (li) por la intensidad de la hipotética interferencia sobre el segundo principio bajo el supuesto de que se omitiera la interferencia con el primero (lj). Esta es, entonces, la fórmula:

$$
W i, j=l i \mid l j
$$

En los casos en los que el valor es mayor que 1 el principio $P i$ precede a $P j$, en los casos en que es menor que $1, P j$ precede a Pi. El empate se da cuando la división es igual a 1. Los valores numéricos pueden asignarse suponiendo que las interferencias leves equivalen a $2^{0}$, las moderadas a $2^{1}$ y las graves a $2^{2}$. 
Insisto en que se trata del peso concreto, es decir, de la precedencia de un principio sobre otro para un caso individual. Como Alexy afirma (Alexy, 2003b, 440): "Las interferencias son siempre interferencias concretas". Es posible añadir en la fórmula lo que Alexy denomina el peso abstracto de los principios, es decir la importancia en abstracto de un principio sobre otro, al margen de las circunstancias del caso concreto. Esto da como resultado la fórmula siguiente ${ }^{12}$ :

$$
W i, j=l i . W i \mid l j . W j
$$

Según Alexy, esta reconstrucción de la ponderación hace de ella una actividad controlable racionalmente. Mientras la subsunción es un esquema que trabaja con arreglo a las reglas de la lógica, la ponderación trabaja de acuerdo a las reglas de la aritmética.

Veamos, a continuación, tres posibles objeciones a este enfoque alexiano. La primera duda que se me plantea es la siguiente: dado que los pesos abstractos de los principios son independientes de cualquier circunstancia concreta, deberiamos tener a nuestra disposición una asignación de peso abstracto para cada principio que establece un derecho constitucional, deberíamos tener una escala de ordenación abstracta de los derechos. No conozco ninguna escala de este tipo que pueda ser aceptada razonablemente. En mi opinión no es claro qué es lo que debería medir la supuesta escala abstracta de ordenación de los derechos constitucionales: ¿debería medir estados de cosas en los que un derecho es máximamente optimizado mientras el otro no lo es en absoluto?

El segundo punto al que quiero referirme guarda relación con la distinción de Alexy entre interferencias leves, moderadas y graves en los principios constitucionales. Aquí estamos frente a una escala, pero ¿de qué depende la asignación de estos tres conceptos en un caso concreto? Sólo podemos construir escalas ordinales o cardinales cuando estamos en presencia de una propiedad claramente definida, como sucede con el test del rayado para la escala de los minerales: un mineral es más duro que otro si y sólo si el primero puede rayar al segundo y el segundo no puede rayar al primero. La dureza de los minerales nos permite de este modo construir una escala ordinal. No veo cómo podemos hacer lo mismo con la interferencia en los derechos constitucionales, dado que no estamos en posesión de nada semejante al test del rayado, ni siquiera somos capaces de delimitar con claridad de qué propiedad estamos hablando frente a la cual la interferencia pueda ser calificada de leve, moderada o grave. $Y$ como hay varias propiedades que son candidatas plausibles a representar ese papel, podemos generar varias escalas, distintas entre sí.

La tercera y última duda que quiero plantear está relacionada con la insistencia de Alexy en que la operación de ponderación se refiere siempre a un caso individual. Lo que conlleva una concepción que ha sido denominada una concepción ad hoc de la ponderación. Mientras la ponderación en abstracto es una ponderación definicional -es decir, una asignación de peso independiente de las circunstancias-, pero inconcluyente, puesto que de la fórmula de Alexy se deriva que un principio con mayor peso en abstracto puede ser derrotado en concreto por otro con menor peso en abstracto; la ponderación en concreto es siempre ad hoc y ello conlleva que "una sola característica peculiar puede justificar una solución diversa de aquella que se ha atribuido a un caso anterior"13. De este modo, se veda uno de los modos de control racional de las decisiones judiciales: aquel basado en la articulación de dicho tipo de decisiones. Es más, se sustituye un modelo generalista de toma de decisiones, por otro de carácter particularista, una sola propiedad diferente ( $y$, como ha de resultar obvio, si dos casos individuales son diferentes entonces tienen al menos una propiedad diferente) puede comportar una solución diversa para el caso.

En mi opinión, estos tres problemas socavan gravemente el modelo proporcionalista de Alexy, puesto que no permiten ni prever lo que los órganos de aplicación del derecho decidirán en los casos de conflictos entre principios constitucionales que establecen derechos fundamentales, ni menos aún someter a crítica racional y articulada sus decisiones.

A menudo se añade a esta crítica la idea según la cual el juicio que subyace a la asignación de peso a los diversos principios es un juicio totalmente subjetivo $y$, como tal, no controlable racionalmente ${ }^{14}$. Baste decir aquí, aunque no puedo argumentarlo, que no considero que la evaluación de la aplicabilidad de los principios sea una tarea plena-

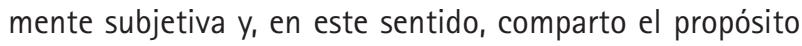
de Alexy de buscar un mecanismo de control racional de esta tarea. 


\section{El ENFOQUe ESPECIFICACIONISTA}

Merece la pena, entonces, explorar el enfoque especificacionista, consistente en reducir el alcance de los principios pero conservar su fuerza. Uno de los puntos centrales en los que insisten los particularistas es que las razones (morales, jurídicas) no se comportan de modo atomista, sino holista; pues bien como ha sido sugerido (Väyrynen, 2006, 710) la estrategia que voy a presentar trata de secuestrar al holismo y acomodarlo en un marco generalista ${ }^{15}$.

Lo haré valiéndome del caso Titanic propuesto tantas veces por Alexy. En este caso se ponen de manifiesto los problemas que he señalado, creo, en la concepción de Alexy. En primer lugar, ¿cuál de los principios, la libertad de expresión y el derecho al honor, tiene mayor peso en abstracto? Nada nos dice al respecto Alexy y, de ello, tal vez haya que concluir que Alexy considera que tienen igual peso. Sin embargo, sólo una teoría plenamente articulada de los derechos nos permitiría alcanzar dicha conclusión y una teoría así está aún por construir. En segundo lugar, ¿por qué denominar al oficial "asesino nato" es una interferencia moderada o leve ( $y$, es más, debería decirse si es moderada o es leve), mientras tildarle de "tullido" es gravísima (que, por cierto, no es una categoría presente en la clasificación de Alexy)? Y ¿por qué imponer una indemnización no muy alta, como en este caso, a los editores de la revista constituye una interferencia grave en la libertad de expresión? Alguien podría argüir, con perfecto sentido, que este tipo de expresiones puede ser evitado sin merma significativa de la libertad de expresión ni de la libertad de información. En tercer lugar, esta sentencia también muestra claramente la dificultad de establecer criterios generales con este método: en el caso de la expresión "asesino nato" la libertad de expresión precede al derecho al honor, en el caso de "tullido" ocurre lo contrario. ¿Qué sucederá, entonces, en otro supuesto de expresión denigratoria en el futuro? ¿Puede alguien decirlo con seguridad?

Por estas razones, creo que es mejor pensar en un modo de configurar la ponderación que la considera un paso previo a la subsunción. Una vía según la cual la ponderación es la operación que permite pasar de las normas que establecen derechos fundamentales, que tienen la estructura de principios - pautas con las condiciones de aplicación abiertas- ${ }^{16}$, a reglas - pautas con las condiciones de aplicación clausuradas-, con las cuales es posible llevar a cabo la subsunción, en el ámbito de un problema normativo determinado. Intentaré mostrar cuáles son las etapas de una operación de este tipo, tomando como supuesto el caso del Titanic.

La primera etapa consiste en la delimitación del problema normativo, de lo que Alchourrón y Bulygin han llamado el universo del discurso ${ }^{17}$. Delimitar claramente el ámbito del problema normativo que nos ocupa permite, principalmente, hacer el problema manejable: ya no nos referimos al conjunto de todas las acciones humanas posibles, sino sólo a un conjunto mucho más reducido de acciones humanas. En el caso del Titanic, el universo del discurso podría ser el siguiente: acciones de información en los medios de comunicación sobre asuntos que afectan a las personas.

La segunda etapa consiste en la identificación de las pautas prima facie aplicables a este ámbito de acciones. Aquí obviamente son aplicables el principio que establece la libertad de expresión e información y el principio que protege el derecho al honor de las personas.

La tercera etapa consiste en la consideración de determinados casos paradigmáticos, reales o hipotéticos, del ámbito normativo previamente seleccionado en la primera etapa. Los casos paradigmáticos tienen la función de constreñir el ámbito de reconstrucciones admisibles: sólo son admisibles aquellas reconstrucciones que reconstruyen los casos paradigmáticos adecuadamente ${ }^{18}$. Los casos paradigmáticos constituyen el trasfondo, a menudo inarticulado, en el cual el razonamiento práctico tiene lugar. En el problema normativo delimitado en la primera etapa a modo de ejemplo, podríamos considerar como paradigmáticos casos como los siguientes: a) publicar la noticia falsa, sin comprobación alguna de su veracidad, de que el arzobispo de Barcelona está implicado en una trama de prostitución infantil, es un ejemplo de un supuesto en el cual la libertad de información cede ante el derecho al honor $y, b$ ) publicar la noticia verdadera de que, por ejemplo, un ministro del gobierno ha cobrado diez millones de euros de cierta empresa a cambio de la concesión para construir una autopista es un supuesto en el que la libertad de información desplaza al derecho al honor.

En la cuarta etapa se establecen las propiedades relevantes de ese universo del discurso. El establecimiento de las propiedades relevantes ha de hacer posible la determinación 
de las soluciones normativas. En nuestro supuesto son claramente relevantes las siguientes propiedades: la relevancia pública de la noticia, que la noticia sea veraz (tal como ello es entendido por muchos altos Tribunales, que sea verdadera 0 , si falsa, diligentemente contrastada) ${ }^{19}$, y que la noticia no sea injuriosa.

La quinta y última etapa consiste en la formulación de las reglas que resuelven de modo unívoco todos los casos del universo del discurso. Una regla, me parece que indiscutida, sería la siguiente:

R1: Las informaciones de relevancia pública, veraces y no injuriosas están permitidas.

También indiscutida, creo, tendríamos una segunda regla:

R2: Las informaciones que no son de relevancia pública o carecen de veracidad o son injuriosas están prohibidas $y$, en el caso que se produzcan, generan un derecho a ser indemnizado.

Obviamente que las tres últimas etapas están íntimamente relacionadas entre sí. El establecimiento de las reglas debe ser controlado de acuerdo con su capacidad de reconstruir los casos paradigmáticos. La selección de las propiedades relevantes debe refinarse en la medida en que este objetivo no sea alcanzado y, a partir de una nueva selección, debe procederse a la formulación del conjunto de reglas que disciplinan dicho problema normativo.

Estas cinco etapas constituyen un modo de concebir la ponderación que lo hace compatible con la subsunción y con una limitada generalidad. Las reglas surgidas de una reconstrucción como la propuesta se aplican de modo subsuntivo y permiten articular y otorgar coherencia a la función judicial, aunque reducen el alcance de los principios. Cuando un órgano jurisdiccional resuelve un caso individual de dicho ámbito normativo, resuelve con su reconstrucción todos los casos individuales de dicho ámbito. Y lo hace de manera compatible con que en otro caso individual perteneciente a ese ámbito, o bien debe seguir la reconstrucción llevada a cabo o debe mostrar una propiedad relevante, no apreciada previamente, que le permita resolver algunos casos individuales de un modo diverso ${ }^{20}$. Creo que de este modo es posible el control racional de la función aplicadora del derecho.
Es obvio que en muchos casos individuales la solución ofrecida por una y otra reconstrucción será la misma. No obstante, insistiré en aquello que las distingue. La concepción aquí defendida requiere que seamos capaces de establecer cuáles son las propiedades seleccionadas relevantes. Guiar el comportamiento es, en realidad, seleccionar propiedades a cuya presencia o ausencia se correlacionan diversas calificaciones deónticas. Obviamente que esta selección de propiedades no está en disposición de resolver todos los casos individuales, principalmente porque los conceptos que describen las propiedades son vagos y siempre quedarán casos de duda: ¿Es determinada expresión un insulto o no, como por ejemplo llamar al oficial de la reserva "asesino nato" en el contexto satírico de la revista Titanic? Esta podría ser una forma de reconstruir la decisión del Tribunal Constitucional alemán con el esquema presentado. La ventaja, en mi opinión, es que el Tribunal en el futuro sólo ha de plantearse si determinada expresión es o no injuriosa y no el grado de interferencia de dicha expresión en la libertad de información.

Por otra parte, en la concepción especificacionista no hace falta la ponderación en abstracto, si por ella entendemos el peso de cada principio al margen de cualquier circunstancia. El derecho a la vida que parece un candidato a tener mayor peso, también está sujeto a especificación: en primer lugar, porque algunas veces el derecho a la vida de una persona entra en conflicto con el derecho a la vida de otra persona, pero también porque aunque el derecho a la vida genera un deber especial de, por ejemplo, los médicos para asistir a aquellos cuya vida corre peligro, dicho deber es exceptuado cuando la persona cuya vida corre peligro ha expresado seria y libremente su rechazo a determinado tratamiento (así las transfusiones de sangre) en virtud de sus convicciones religiosas.

Por último y más importante: el modelo de Alexy está abocado al particularismo, en el sentido de que una propiedad diferente puede hacer que un nuevo caso tenga una solución distinta. El modelo especificacionista no está necesariamente vinculado con el particularismo, en un ámbito determinado y con determinados principios en liza, el modelo presentado es generalista y con él se resuelven todos los casos previamente delimitados.

Considero la estrategia especificacionista razonable. Sin embargo, para que dicha estrategia sea convincente debe 
ser sometida a dos limitaciones importantes ${ }^{21}$. Por una parte, dicha especificación contendrá siempre, entre las circunstancias que permiten revocar la obligación moral a la que en principio llevan determinadas propiedades -llamémosle defeaters-22, conceptos morales. Supongamos que yo he prometido ir esta noche a cenar a casa de un amigo. Si mi amigo me llama para decirme que si no termino el artículo que estoy escribiendo puedo no ir a su casa, este hecho socava la razón para cumplir la promesa, se trata de un underminer, una causa de supensión. Ahora bien, si mi amigo me llama para decirme que está muy cansado y que no se siente con fuerzas para cocinar esta noche, entonces este hecho cancela mi obligación de un modo diverso al anterior: no sólo la socava sino que me ofrece una razón para no ir a su casa, se trata de un reverser, una causa de inversión. Y si yo sufro mareos y no me encuentro bien para ir a su casa, entonces mi responsabilidad es anulada mediante una excusa. Por otra parte, si es mi mujer la que me llama desde el hospital en el que ha sido ingresada, entonces el deber de acudir al hospital revoca el deber de cenar con mi amigo, se trata de un overrider, una causa de anulación. Como dice Walter Sinnot-Armstrong, al que se deben estas ideas y esta terminología: "Overriders, underminers, reversers, and excuses are, then, all reason defaters" (anulaciones, suspensiones, inversiones y excusas son, entonces, todas ellas causas de revocación de las razones) ${ }^{23}$.

Entonces, los principios morales deben ser formulados incluyendo sus defaters. Ahora bien, los defeaters siempre contendrán conceptos morales. Sólo en este sentido es posible codificar los principios morales. Es claro que, al menos, por razones epistémicas no podemos codificar todos los defeaters acudiendo únicamente a propiedades naturales. Además, dadas las infinitas descripciones posibles de las acciones, es plausible pensar que también dicha tarea es irrealizable por razones conceptuales ${ }^{24}$. Este es el sentido en el cual el universalismo puede ser adecuado: hay principios morales, ahora bien -y esta es la concesión al particularismo- que contienen necesariamente conceptos evaluativos ${ }^{25}$. Podemos de este modo revisar los antecedentes de nuestros principios prima facie haciéndolos más específicos, pero con el coste de incorporar en ellos, como defeaters, conceptos evaluativos.

Así es como, por otra parte, están codificadas las normas jurídicas. Si alguien mata a otro, comete un homicidio cas- tigado, en principio, por el código penal; pero dicha acción no es punible si ha sido realizada en legítima defensa o en estado de necesidad o cualquiera del resto de causas de justificación, o ha sido realizada en presencia de alguna de las causas de exculpación (trastorno mental, por ejemplo). Las causas de justificación y las excusas son los defeaters en la codificación de las normas penales. Dichos defeaters están de hecho formulados de tal modo que incorporan conceptos evaluativos (en el caso de la legítima defensa, que la agresión a la que se responde sea ilegítima, que no mediare provocación suficiente, etc.) Si alguien realiza un contrato de compraventa, obligándose a la entrega de un coche a cambio de un precio, tal contrato es válido siempre que no medien, por ejemplo, algunos vicios del consentimiento. Es decir las obligaciones en el derecho privado están también sujetas a defaters: los contratos son nulos si son contrarios a las leyes, a la moral o al orden público, son también nulos si se otorgan con algún vicio del consentimiento (error, coacción, etc.). Como puede apreciarse, también en este caso los defeaters contienen conceptos evaluativos.

No veo razones para rechazar este punto de vista para la moral, ni desde el punto de vista conceptual, ni desde el punto de vista justificativo. Ni siquiera Jonathan Dancy rechaza esta posibilidad ${ }^{26}$. Ahora bien, Dancy replica diciendo que de este modo no conseguimos todavía dar forma (shape) a las propiedades naturales sobre las que las propiedades morales supervienen, puesto que ahora queda por averiguar sobre qué propiedades naturales supervienen los defaters. Es decir, esta forma de universalismo no es más que un particularismo disfrazado de universalismo, un lobo con piel de cordero. Es un argumento similar al de Bruno Celano ${ }^{27}$. Celano sostiene que no hay ningún modo estable de llevar a cabo la revisión desde nuestros condicionales derrotables hasta los condicionales inderrotables y no lo hay porque la noción de una tesis de relevancia última está mal formada. La tesis de relevancia última es aquella que establece cuáles son las propiedades naturales relevantes de un modo exhaustivo ${ }^{28} \mathrm{y}$, según Celano, no hay esperanzas de alcanzar tal objetivo. Según Celano, el conjunto de las combinaciones de las propiedades potencialmente relevantes, que toman en cuenta la historia del caso, no es agotable ${ }^{29}$.

Estas consideraciones de Celano (y de Dancy) nos llevan a otra limitación del universalismo. Concedo, sin más ar- 
gumentos, que en la codificación de las normas morales (y jurídicas) no puede irse más allá de principios o normas con defaters evaluativos. Sin embargo, el discurso de aplicación de dichas codificaciones puede ir más allá. Sería irrazonable suponer que, enfrentados a un problema moral, debamos reconstruir todos nuestros principios morales válidos para todas las acciones humanas posibles. Es más sensato considerar que podemos adoptar un determinado universo del discurso, es decir, un conjunto determinado de acciones humanas. Por ejemplo, el conjunto de las acciones de devolver los libros que tenemos en préstamo ${ }^{30}$. Tal vez en este caso, podamos alcanzar algo como una tesis de relevancia. Son relevantes, con seguridad, el plazo de tiempo por el que tenemos el préstamo, el hecho de que el que nos lo prestó sea su verdadero propietario (no es un libro sustraído de la biblioteca universitaria, por ejemplo), debemos incluir también las justificaciones (tuvimos que ausentarnos de la ciudad por un tiempo) y las excusas (lo dejamos olvidado en un cambio de domicilio). Podemos también delimitar previamente el conjunto de normas morales a tomar en cuenta, para ignorar casos recalcitrantes siempre imaginables. Podemos atender únicamente a la norma moral que obliga a devolver lo prestado bajo ciertas condiciones e ignorar, ahora, la norma moral que prohíbe inducir a otro al suicidio, y así ignorar el supuesto de que la lectura del libro, dado el estado anímico de su propietario, puede llevarlo a suicidarse. Obviamente que si apreciamos que este es el caso, debemos introducir esta norma en el sistema. Pero limitada a un universo de acciones y a un subconjunto de las normas morales, creo que es razonable aspirar a obtener una tesis de relevancia manejable. Por otro lado, así operan los juristas cuando se enfrentan a un caso real o hipotético: toman en cuenta un universo del discurso delimitado y un subconjunto de normas jurídicas (no todas las normas jurídicas, ya que no es sensato, aunque son un conjunto finito -si nos limitamos a las normas formuladas y no tomamos en cuenta las derivadas-, pensar en una reconstrucción global de todas ellas). Para micro- sistemas así concebidos la operación de revisión estable es alcanzable y permite pasar, en este contexto delimitado, de la formulación de defeaters evaluativos a defeaters descritos mediante propiedades naturales. Obviamente que se trata, por definición, de reconstrucciones incompletas. También es verdad, como quieren los particularistas, que dichas reconstrucciones dependen del contexto, pero no dependen -como arguyen los particularistas- del contexto establecido por las circunstancias del caso concreto, sino que dependen del contexto del problema normativo seleccionado. Es decir, en un contexto normativo así delimitado, hay propiedades naturales uniforme e invariablemente relevantes.

\section{Frónesis aristotéLICA PARA CONCLUIR}

Alguien puede considerar, con razón, que esta defensa del generalismo concede demasiado al particularismo, dado que sólo tenemos principios morales si los concebimos como incorporando conceptos evaluativos y en el razonamiento práctico el juego de estos principios está delimitado por el contexto del universo del discurso seleccionado y de los principios morales que consideramos aplicables. Sin embargo, aún con estos límites, la imagen del razonamiento práctico que obtenemos permite la subsunción de casos individuales en pautas generales y deja un razonable espacio para la articulación y la consistencia de las decisiones. Esta concepción del razonamiento práctico vale, en mi opinión, tanto para la moral cuanto para el derecho.

Quiero decir, para terminar, que me complacería que las ideas presentadas aquí sean sólo un desarrollo de la concepción aristotélica de la deliberación práctica, de la frónesis aristotélica ${ }^{31}$. El cómo y el porqué deberán, me temo, aguardar a otra ocasión.

Recibido: 21 de enero de 2009

Aceptado: 23 de febrero de 2009 
1 BverfGE vol. 86, 1.

2 Vid. STC 20/1992, de 14 de febrero.

3 En la filosofía contemporánea representadas por, por ejemplo, Dancy, 1993, 2004; McNaughton, 1988, y McDowell, 1998.

4 En virtud, digamos, del principio moral que nos obliga a no mentir. Kant pensaba que tal principio es un principio categórico, absoluto y por ello escribió (1989, 292): "La mentira (en el sentido ético de la palabra), como falsedad deliberada, no precisa perjudicar a otros para que se la considere reprobable... Su causa pude ser la ligereza o la bondad, incluso puede perseguirse con ella un fin realmente bueno, pero el modo de perseguirlo es, por la mera forma, un delito del hombre contra su propia persona y una bajeza que tiene que hacerle despreciable a sus propios ojos".

5 Éstas parecen ser las razones que conducen a algunos autores a rechazar la tesis de los conflictos entre los derechos y a defender alguna versión del enfoque universalista y subsuntivo. Vid., por ejemplo, Habermas, 1992, cap. 6 y Ferrajoli, 2001, 353-354.

6 En Dancy, 2004, 3-12, se halla la misma idea como "rango de opciones" y la presentación de algunas de ellas.

7 Shafer-Landau, 1995a, 225.

8 Alexy, 1986, 2002, 2003a, 2003 b.

9 Dancy (Dancy, 2004, 11) llama a esta posición "expansionismo".

$10 Y$ que se inspira en algunas ideas de Hare, 1952, 48-55, 60-65; Hurley, 1989, 1990; Richardson, 1990; Bayón, 2001; Atienza-Ruiz Manero, 2002, y Scanlon, 2000. Con atención especial al particularismo y al modo en que una posición como esta puede preservar un espacio conceptual para los principios generales, vid. Sinnot-
Amstrong, 1999; Holton, 2002, y Väyrynen, 2006.

11 Estas dos operaciones deben ser comprendidas como distintas en un sentido sólo heurístico y no conceptual. Quiero decir que, conceptualmente, si un medio A es necesario para obtener $B$ también es adecuado. Ahora bien, según creo, hay que interpretar a Alexy en el sentido siguiente: en primer lugar, averiguamos si A se encuentra entre los medios adecuados para obtener $B$ y, en segundo lugar, vemos si no existen medios menos lesivos que $A$ de alcanzar $B$.

12 En realidad, Alexy añade otra complicación en la fórmula, de la que aquí prescindiré, relativa a la confiabilidad de las asunciones empíricas concernientes a las diversas interferencias en juego. Alexy, 2003b, 446-448.

13 Bernal Pulido, 2003, 188-189, en la mejor presentación y desarrollo de las ideas de Alexy en castellano. Para la distinción entre ponderación definicional y ponderación ad hoc vid. por ejemplo Aleinikoff, 1987, 948.

14 Por ejemplo, Guastini, 1999, 169171; también, 1998a, 1998b, 302304; Hernández Marín, 2005, 322327, y García Amado, 2007a, 2007b. Lo que parece incomodar a García Amado, especialmente, es el aumento del poder de control jurisdiccional de los tribunales constitucionales frente a los tribunales ordinarios, a su -como dice- "tácito cometido como superapelación". Aunque este punto exigiria un tratamiento más detallado, baste decir aquí que si los derechos constitucionales han de ser efectivos, alguien deberá velar por ellos $y$, me temo, que durante estos treinta años de democracia constitucional en España, si no lo hubiese hecho el Tribunal Constitucional, algunos de nuestros derechos habrian quedado desprotegidos. Más extraña, me parece, la posición de Hernández Marín, a la que tampoco puedo prestar la atención que merece, puesto que sostiene que -sea o no subjetivo el juicio de ponderación- el juez cumple con el derecho aplicando cualquiera de los dos principios en pugna. Tal vez habría que revisar la noción de aplicación y de cumplimiento del derecho que permite esta extraña conclusión, a saber, que el juez del caso Titanic, por ejemplo, cumple juzgando que se debe rechazar la demanda del militar porque las publicaciones están protegidas por la disposición constitucional que protege la libertad de expresión y cumple también admitiendo la demanda porque se ha vulnerado la disposición constitucional que protege el derecho al honor.

15 La he desarrollado previamente en Moreso, 2002, 2004.

16 Para esta noción de principios vid. Ateinza-Ruiz Manero, 1996, cap. 1.

17 Alchourrón-Bulygin, 1971, cap. I.

18 Vid., para esta función de los casos paradigmáticos en el ámbito del razonamiento jurídico, Dworkin, 1986, 255-257; Hurley, 1989, 212, y Endicott, 1998.

19 Se trata de la doctrina del Tribunal Supremo de los Estados Unidos en New York Times v. Sullivan 376 US 254 (1964), en donde se aplica el denominado "malice test": "knowledge of falsehood or reckless disregard for falsity", recogida por muchos Tribunales Constitucionales europeos. Para España [que, por otra parte, en el propio texto constitucional, en el art. 20.1.d), reconoce explicítamente el derecho a "comunicar o recibir libremente información veraz..."] pueden verse, por ejemplo, las siguientes sentencias del Tribunal Constitu- 
cional: STC 6/1988, de 21 de enero, STC 40/1992, de 30 de marzo y STC 240/1992, de 21 de diciembre.

20 Como es obvio esta operación representa el cambio de las reglas del sistema normativo $y$, por lo tanto, del sistema normativo aplicable. Sin embargo, este cambio es compatible con el hecho de que la solución de los casos realmente ocurridos en el pasado sea la misma para los dos sistemas normativos. Vid. el desarrollo de esta idea en Moreso, 2002.

21 Expongo algunas ideas ya presentes en Moreso, 2005b.

22 Vid. Moreso, 2005a.

23 Sinnot-Amstrong, 1999, 5.

24 Dicha sugerencia en Celano, 2007 y Moreso, 2004a (ahora cap. 18).

25 Esta es la posición de McNaughtonRawling, 2000.

26 Dancy, 1999, 2001.

27 Celano, 2007.

28 La noción de tesis de relevancia se halla en Alchourrón-Bulygin, 1971, cap. VI. Vid. también Rodríguez-Súcar, 1998, y Rodríguez, 2000.

29 Celano, 2007.

30 Por tomar un ejemplo de Dancy, 1993, 60.

31 En la línea, por ejemplo, del cap. 6 de Wiggins, 1987, o del cap. 10 de Nussbaum, 2001.

\section{BIBLIOGRAFÍA}

Alchourrón, Carlos E.; Bulygin, Eugenio (1971): Normative Systems, New York-Wien, Springer.

Aleinikoff, T. Alexander (1987): "Constitutional Law in the Age of Balancing," Yale Law Journal, 96, 943-1005.

Alexy, Robert (1986): Theorie der Grundrechte, Frankfurt, Srukhamp, trad. de E. Garzón Valdés, Teoría de los dere- chos fundamentales, Madrid, Centro de Estudios Constitucionales, 1993.

- (2002): "Epílogo a la Teoría de los derechos fundamentales", trad. de C. Bernal, Revista española de Derecho Constitucional, 22, n. ${ }^{\circ} 66,13-64$.

- (2003a): "Constitutional Rights, Balancing, and Rationality", Ratio luris, 16, 131-140.

- (2003b): "On Balancing and Subsumption. A Structural Comparison", Ratio luris, 16, 433-449.

Atienza, Manuel y Ruiz Manero, Juan (1996): Las piezas del Derecho, Barcelona, Ariel.

- (2000): "Rules and Principles Revisited", Associations, 4, 147-156.

Bayón, Juan Carlos (2001): "¿Por qué es derrotable el razonamiento jurídico", Doxa, 24, 35-62.

Bernal Pulido, Carlos (2003): El principio de proporcionalidad y los derechos fundamentales, Madrid, Centro de Estudios Políticos y Constitucionales.

Celano, Bruno (2007): "Pluralismo etico, particolarismo e caraterizzazioni di desiderabilità: il modello triadico", Ragion Pratica, 1 (giugno), 133-150.

Dancy, Jonathan (1993): Moral Reasons, Oxford: Basil Blackwell.

- (1999): "Defending Particularism", Metaphilosophy, 30, 24-32.

- (2000): Practical Reality, 0xford, Oxford University Press, 2000.

- (2001): "Moral Particularism", Edward N. Zalta (ed.), The Stanford Encyclopedia of Philosophy, Summer 2001 edition, http://plato.stanford.edu/archives/ sum2001/entries/moral-particularism/.

- (2004): Ethics without Principles, Oxford, Oxford University Press.

Dworkin, Ronald (1986): Law's Empire, Cambridge, Mass., Harvard University Press.

Endicott, Timothy (1998): "Herbert Hart and the Semantic Sting", Legal Theo$r y, 4,283-301$.
Ferrajoli, Luigi (2001): Los fundamentos de los derechos fundamentales, Madrid, Trotta.

García Amado, Juan Antonio (2007a): "El juicio de ponderación y sus partes. Una crítica," en R. Alexy, Derechos sociales y ponderación, ed. a cargo de Ricardo García Manrique, Madrid, Fundación Coloquio Jurídico Europeo, 2007, cap. VII.

- (2007b): “¿Ponderación o simples subsunciones? Comentario a la Sentencia del Tribunal Constitucional Español 72/2007, de 25 de abril de 2007", Palestra del Tribunal Constitucional, Revista mensual de jurisprudencia, Lima, 2, n. ${ }^{\circ}$ 8, 619-627.

Guastini, Riccardo (1998a): "Principi di Diritto e discrezionalità giudiziale", Diritto Publico, 651-659.

- (1998b): Teoria e dogmatica delle fonti, Milano, Giuffrè.

- (1999): Distinguiendo, Estudios de teoría y metateoría del Derecho, trad. castellana de J. Ferrer, Barcelona, Gedisa.

Habermas, Jürgen (1992): Facticidad y validez. Sobre el Derecho y el estado democrático de derecho en términos de teoría del discurso, trad. de M. Jiménez Redondo, Madrid, Trotta.

Hare, R. M. (1952): R. M. The Language of Morals, Oxford, Oxford University Press.

Hernández marín, Rafael (2005): Las obligaciones básicas de los jueces, Madrid, Marcial Pons, 2005.

Holton, Richard (2002): "Principles and Particularisms", Proceedings of the Aristotelian Society, suppl., vol. 76, 191-210.

Hurley, Susan (1989): Natural Reasons, Oxford, Oxford University Press.

- (1990): "Coherence, Hypothetical Cases, and Precedent", Oxford Journal of Legal Studies, 10, 221-255.

Kant, Immanuel (1989): La metafísica de las costumbres, trad. de A. Cortina Ors y J. Conill Sancho, Madrid, Tecnos. 
McDowell, John (1998): Mind, Value, and Reality, Cambridge, Mass.; Harvard University Press.

McNaughton, David (1988): Moral Vision, Oxford, Oxford Blackwell.

McNaughton, David y Rawling, Piers (2000): "Unprincipled Ethics," en H Hooker, Brad y Little, Margaret (eds.) (2000), Moral Particularism, 0xford, Oxford University Press.

Moreso, José Juan (2002): "Conflitti tra principi constituzionali", Ragion Pratica, 18, 201-221.

- (2004): "Dos concepciones de la aplicación de las normas de derechos fundamentales," en Betegón, Jerónimo; Laporta, Francisco; Páramo, Juan Ramón, y Prieto Sanchís, Luis (comps.) (2004): Constitución y derechos fundamentales, Madrid, Centro de Estudios Políticos y Constitucionales.
- (2005a): "Positivismo giruidico e applicazione del diritto", Materiali per una storia della cultura giuridica, 35, 225244.

- $\quad$ (2005b): "Cristina Redondo su ragione e norme", Ragion Pratica, 2 (dicembre), 491-506.

Nussbaum, Martha C. (2001): The Fragility of Goodness: Luck and Ethics in Greek Tragedy and Philosophy, 2 edition, Cambridge, Cambridge University Press.

Richardson, H. S. (1990): "Specifying Norms as a Way to Resolve Concrete Ethical Problems", Philosophy and Public Affairs, 19, 279-310.

Rodríguez, Jorge L.(2000): "Axiological Gaps and Normative Relevance," Archiv für Recths-und Sozialphilosophie, 86, 151-167.

Rodríguez, Jorge L.; SÚCAR, Germán (1998): "Las trampas de la derrotabilidad. Niveles de análisis de la indetermina- ción del derecho," en Analisi e Diritto, 1998, 277-305.

Ross, W.D. (1930): The Right and the Good, Oxford, Oxford University Press.

Scanlon, Thomas (2000): "Intention and Permissibility", Proceedings of the Aristotelian Society, suppl., vol. 74, 301-317.

Shafer-Landau, Russ (1995): "Specifying Absolute Rights", Arizona Law Review, 37, 209-225.

Sinnot-Armstrong, Walter (1999): "Some Varieties of Particularism", Metaphilosophy, 30, 1-12.

Väyrynen, Pekka (2006), "Moral Generalism: Enjoy in Moderation", Ethics, 116, 707-741.

Wiggins, David (1987): "Deliberation and Practical Reason", en D. Wiggins, Needs, Values, and Truth. Essays in the Philosophy of Value, Oxford, Basil Blackwell, 1987. 